\title{
Calibrating Sensors to Measure Braking Chopper Currents in DC Traction Units
}

This paper was downloaded from TechRxiv (https://www.techrxiv.org).

\section{LICENSE}

CC BY 4.0

SUBMISSION DATE / POSTED DATE

$21-12-2020$ / 30-12-2020

CITATION

van den Brom, Helko; van Leeuwen, Ronald (2020): Calibrating Sensors to Measure Braking Chopper Currents in DC Traction Units. TechRxiv. Preprint. https://doi.org/10.36227/techrxiv.13469679.v1

$\mathrm{DOI}$

10.36227/techrxiv.13469679.v1 


\title{
Calibrating Sensors to Measure Braking Chopper Currents in DC Traction Units
}

\author{
Helko van den Brom and Ronald van Leeuwen \\ VSL, Delft, The Netherlands \\ hvdbrom@vsl.nl
}

\begin{abstract}
A large part of the energy involved in the braking stage of conventional DC railway traction units is dissipated in rheostat resistors, which is controlled by means of a braking chopper. A setup was developed to calibrate transducers used to measure the resulting high-current chopped DC current signals under conditions mimicking the real application. Preliminary results indicate that the reference transducer used in this setup can accurately measure chopped signals with magnitudes up to at least $250 \mathrm{~A}$ and pulse repetition rates up to $500 \mathrm{~Hz}$ with uncertainties better than $0.01 \%$.
\end{abstract}

Index Terms - Current measurement, measurement standards, measurement techniques, precision measurements, transducers.

\section{INTRODUCTION}

A significant amount of energy used by DC railway traction units is wasted during the braking process. When braking, the traction motor is converting mechanical energy of the rotating shaft to electrical energy, which is returned to the overhead line. Since DC overhead lines are usually supplied by unidirectional AC to DC substations, the DC voltage will increase. Above a specified voltage level, an on-board braking chopper will be activated, mostly for dissipation of the energy in braking rheostat resistors. Railway companies are investigating how to recover this energy, for example by installing bidirectional reversible substations or on-board storage systems such as batteries or supercapacitors.

The amount of dissipation is controlled by means of pulsewidth or frequency modulation of the braking chopper on-off switch. The resulting current signal varies between zero and maximum, typically with switching frequencies of hundreds of hertz. This current signal can be measured using sampling techniques, but the accuracy is limited because the behavior of the transducers is not known for such chopped signals.

In this paper preliminary results of the development of a reference setup are presented for the calibration of current transducers using chopped high-current waveforms for braking chopper applications in DC traction units. The setup is based on a current ratio measurement system [1] that was modified for the characterization of DC current sensors in the presence of $\mathrm{AC}$ distortion developed for railway applications [2].

\section{MEASUREMENT SETUP}

The operating principle of the measurement setup is to send a chopped DC current through the current transducer under test and a reference transducer in series and to measure the ratio of the respective output voltage signals $V_{\text {out }}$ by means of two synchronized sampling voltmeters. The transresistance error of the device under test is then defined as

$$
\varepsilon_{d u t}=1-\frac{R_{\text {nom }}^{\text {dut }}}{R_{\text {nom }}^{\text {ref }}} \cdot \frac{V_{\text {out }}^{\text {ref }}}{V_{\text {out }}^{\text {dut }}}+\varepsilon_{\text {ref }},
$$

where $R_{\text {nom }}$ is the nominal transresistance ratio of the device under test and the reference transducer, respectively.

The chopped waveforms are generated using a 900 A highpower DC current source in combination with a programmable DC electronic load that can define load profiles with currents up to $600 \mathrm{~A}$ and a bandwidth of $20 \mathrm{kHz}$.

The ratio of the output voltages of the two transducers is measured using two high-precision digital voltmeters with a sampling rate of $50 \mathrm{kS} / \mathrm{s}$. The voltage ratio can be determined with an accuracy of better than two parts in $10^{6}[1,2]$.

The reference transducer consists of a high-precision zeroflux current transformer combined with a broadband current shunt to convert the output current to voltage. The zero-flux transformer has a nominal ratio of 1500:1, a maximum primary current of $900 \mathrm{~A}$, and a bandwidth of $1 \mathrm{MHz}$. The $1 \mathrm{~A}$ current shunt was designed to have very low AC-DC difference for frequencies up to $100 \mathrm{kHz}$. The total uncertainty of the combined reference transducer was found to be better than $10 \mu \Omega / \Omega(k=2)$ in the presence of AC distortions [1]. For the application discussed in this paper, however, the reference transducer must be proven to accurately convert high-current DC chopped input signals into identical voltage waveforms, and the sampling ratio measurement system must be proven to accurately determine the correct ratio for these signals as well.

\section{EXPERIMENTAL RESULTS}

\section{A. Measurements with reference transducer and shunt}

In previous work, the setup was used to compare a commercial DC current shunt with the previously mentioned reference transducer. As a proof-of-principle, we used the same approach and programmed the electronic load to simulate an ideal chopped DC waveform switching between $0 \mathrm{~A}$ and $250 \mathrm{~A}$ with a frequency of $500 \mathrm{~Hz}$. The waveform programmed to the electronic load and the signals measured using the reference transducer and the current shunt under test are presented in figure 1. Obviously, both the current measured by the reference transducer and the shunt do not properly reflect the behavior of the intended input current. The reference transducer only seems 


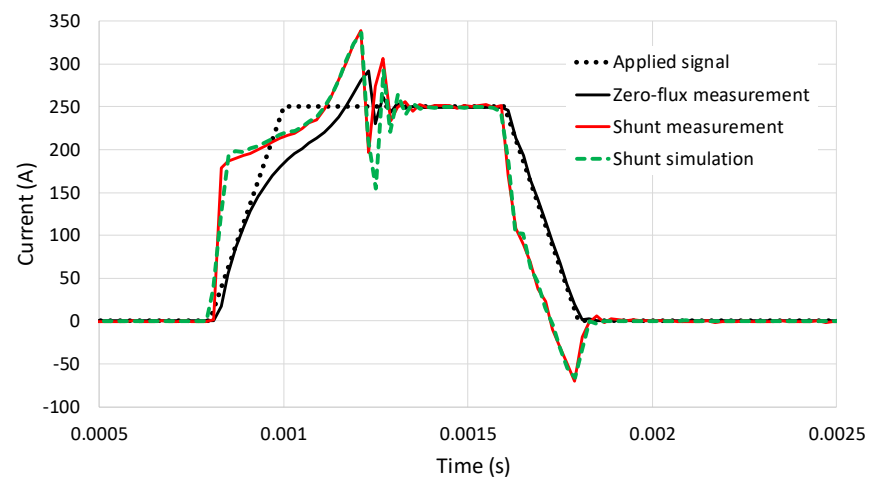

Fig. 1. Measurements using the zero-flux transducer and the current shunt compared to the applied signal and to simulation of the shunt.

to accurately follow the applied current signal and thus to meet our expectations during the falling edge.

To improve the current signal, the power supply output was modified by means of a parallel capacitor. Figure 2 shows that when increasing the capacitance, the intended $1 \mathrm{kHz}$ chopped waveform as measured using the reference transducer was better resembled. However, below $150 \mathrm{~A}$, the rise time hardly changed, which might be due to the limited slew rate of the programmable load. Therefore, other methods need to be investigated to generate the desired chopped waveforms.

\section{B. Simulations of the shunt}

For further investigation, we modeled the shunt as a resistor with a series inductor and a parallel capacitor. The values of the components involved were optimized to find a good match between the simulated current and the measured output signal of the shunt. However, whereas a very good match could be found for the falling edge part of the signal, a huge mismatch on the rising edge remained unsolved.

When using the current measured with the reference transducer as applied current in the simulations, however, we obtained the results presented in figure 1. In this case the measurement and the simulation follow an almost identical waveform on both edges.

\section{DISCUSSION}

The different reference and shunt measurements can be well understood for the falling edge but not for the leading edge of the chopped input waveform. Hence, during the rising edge, the real input waveform deviates from the intended waveform. Apparently, the source cannot deliver the requested current sufficiently fast, whereas reducing the current from maximum to zero is much easier to establish. The parallel capacitor that improved the rising edge can be understood to quickly provide the necessary amount of charge, after which the source gradually takes over and recharges the capacitor.

If the reference transducer is assumed to measure correctly during both falling and rising edge, the measured current must

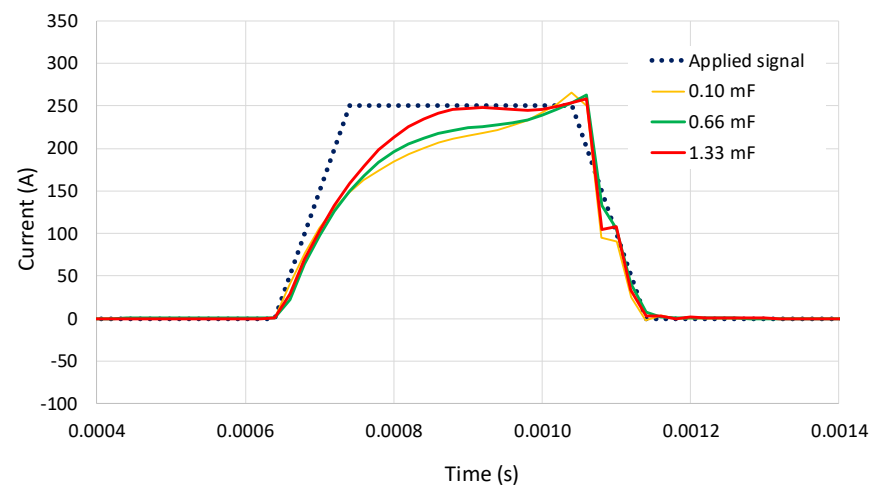

Fig. 2. Current signals as generated using different values for the parallel capacitor, as measured using the reference transducer.

be applied as input current in the simulations. Indeed, when doing so, the measurement and simulation follow an almost identical curve on both edges. This justifies our assumption regarding the bias source failing on the rising edge and the reference transducer accurately following the input signal.

\section{CONCLUSION AND FUTURE WORK}

To facilitate the accurate measurement of high-current chopped DC currents, as typically occurring during the braking process of traction units, a setup to characterize DC current transducers for railway applications was further improved. Measurements and simulations showed that the reference transducer accurately follows the chopped waveform for magnitudes and repetition rates up to at least $250 \mathrm{~A}$ and $500 \mathrm{~Hz}$, respectively. Since the accuracy of the transducer is better than $10 \mu \Omega / \Omega$ in the presence of AC distortion, measurements of chopped waveforms with uncertainties better than $0.01 \%$ are within reach. However, the current generation part of the setup still needs further improvement. Furthermore, higher currents are desired to meet real application demands.

\section{ACKNOWLEDGEMENT}

The research leading to the results described in this paper is performed within the 16ENG04 MyRails project of the European Metrology Programme for Innovation and Research (EMPIR). The EMPIR initiative is co-funded by the European Union's Horizon 2020 research and innovation programme and the EMPIR participating states.

\section{REFERENCES}

[1] H. E. van den Brom, G. Rietveld, and E. So, "Sampling Current Ratio Measurement System for Calibration of Current Sensors up to $10 \mathrm{kA}$ with $5 \cdot 10^{-6}$ Uncertainty", IEEE Trans. Instr. Meas, vol. 64, no. 6, pp. 1685-1691, June 2015.

[2] H. E. van den Brom, R. van Leeuwen, and R. Hornecker, "Characterization of DC current sensors with AC distortion for railway applications", IEEE Trans. Instrum. Meas., Vol. 68, pp. 2084-2090, June 2019. 